\title{
SLM-based educational kit for wave optics
}

\section{Chen Wang, Yu-Chia Hsu, Sheng-Hsiung Chan}

Chen Wang, Yu-Chia Hsu, Sheng-Hsiung Chan, "SLM-based educational kit for wave optics," Proc. SPIE 9793, Education and Training in Optics and Photonics: ETOP 2015, 979315 (21 January 2016); doi: 10.1117/12.2223100

SPIE Event: Education and Training in Optics and Photonics: ETOP 2015, 2015, Bordeaux, France 


\title{
SLM-Based Educational Kit for Wave Optics
}

\author{
Chen Wang, Yu-Chia Hsu, Sheng-Hsiung Chan \\ Jasper Display Corp. TW 7F-16, No. 81, Shuili Rd., East Dist., Hsinchu City 30059, Taiwan \\ (R.O.C.), +886-3-575-2768, info@jasperdisplay.com
}

\begin{abstract}
Spatial Light Modulator (SLM) is a powerful active optical component to change the optical wavefront by electrical signal and had been widely investigated for many applications. We use LCoS (Liquid Crystal on Silicon) which has high reflectivity and spatial resolution as main component of SLM educational kit, and present a series of experiment to let students know the background theory of SLM. Our course mainly has three parts, the first part is the principle of LCoS SLM which has polarization, jones matrix, and uniaxial crystal theory. The second part is wave optics which can see interference, diffraction, and dispersion phenomenon. The last part is Fourier optics which has the concept of spatial frequency, spectrum, Fresnel diffraction, and Fraunhofer diffraction. This educational kit also includes software that produces some basic diffraction grating which dynamically response to parameter changing and customized user's own diffraction grating by macro commands. In this paper we will describe the background theory, experiments design, and result of experiments to show what students can learn from the SLM educational kit.
\end{abstract}

Keywords: Spatial Light Modulator, Educational kit, Wavefront modulation

\section{INTRODUCTION}

Recent years have seen increased attention being given to Liquid Crystal SLM's applications e.g. adaptive optics ${ }^{1}$, holographic display ${ }^{2}$, beam shaping ${ }^{3}$, and beam steering ${ }^{4}$. There are two type of LC-SLM: transmissive type and reflective type. Reflective type SLM typically has more efficient light usage and less annoying diffraction by high aperture ratio. Consequently we decide to design a series of experiments which are sorted into 3 topics for reflective type LC-SLM, and each of these topics has 3 to 4 experiments.

The first topic, "Fundamentals of SLM", is designed to let students familiar with reflective type LC-SLM. It has four experiments regarding how pixelated structure diffracts light ${ }^{5}$, how to use SLM as an amplitude/phase modulator ${ }^{6}$, and how to modulate the wavefront ${ }^{7}$. After completing these experiments, students have had the background knowledge to operate SLM system including SLM and optical components setup for SLM. Next we try to apply the SLM to traditional optical experiments, single/double slit diffraction ${ }^{7}$, Michelson interferometer ${ }^{7}$, and dispersion ${ }^{7}$. The SLM is not only a wavefront modulator but also a programmable mask, we can change any mask setting like slit width, separation of two slits, and pattern period, then we can easily see the difference of experiment result. At the last topic, we'll go further into Fourier optics which discusses the optical wave propagates in space by Fourier analysis. There are three experiments related to Fourier optics, signal processing ${ }^{5}$, Talbot image ${ }^{5}$, and phase shifting digital holography ${ }^{5}$. SLM can provide fine grating with 1080p resolution for these experiments.

SLM is a powerful tool for light wave modulation and related applications but it highly needs theoretical knowledgement to operate it, especially for holographic applications. Therefore, we're trying to promote it by a serious of experiments which only needs basic diffraction patterns, and we also provide GUI to easily draw these patterns for students.

\section{REFLECTIVE SPATIAL LIGHT MODULATOR}

LCoS-SLM has pixelated structure as micro display. When coherent light source illuminates the pixelated structure, it is diffracted both in vertical and horizontal direction, and the polarization state is changed by the anisotropic characteristic of liquid crystal. Using this characteristic of LC, SLM can modulate amplitude or phase of light. Our goal of the first section is that let students know these characteristics of SLM and use it to modulate basic wavefront.

The main optical system for reflective SLM in our educational kit is shown in Fig. 1. Most of the experiments are under this configuration but has slight difference in each experiment. The first part of our optical system (purple) from Laser to collimating lens is collimating system which produces a plane wave to illuminate the SLM. The second part is

Education and Training in Optics and Photonics: ETOP 2015, edited by Eric Cormier, Laurent Sarger Proc. of SPIE Vol. 9793, 979315 - @ 2015 SPIE, IEEE, OSA, ICO · doi: 10.1117/12.2223100 


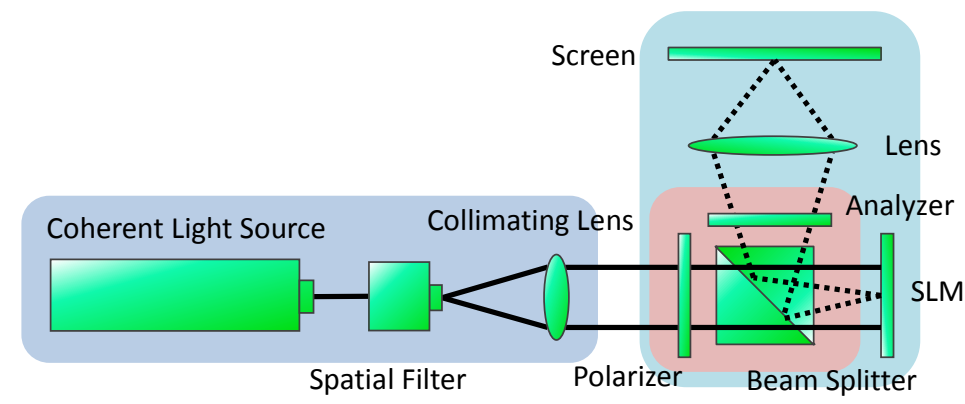

Fig. 1. Basic setup for LCoS SLM.

polarization system (red), two polarizers constrain the input and output polarization state to switch SLM between amplitude modulator and phase modulator. The last part is an imaging system (blue), the imaging lens will form SLM image onto screen or CCD. Our educational kit for SLM mainly uses these three parts to compose ten experiments.

We have four experiments in the first section. At the first experiment, we tried to measure the period of the SLM pixelated structure. The experiment setup is shown in Fig. 2(a), with a simple illuminating configuration for reflective type SLM, we only keep the polarization part and two polarizers are aligned with ordinary axis of liquid crystal to prevent from variation in index of refraction. Laser normally incident on SLM and then it is reflected back with many diffraction orders as shown in Fig. 2(b). By Fourier series analysis, a periodical structure can be expressed by following equation

where

$$
f(x)=\sum_{-\infty}^{\infty} A_{n} e^{j 2 \pi \frac{n}{D} x},
$$

$$
A_{n}=\frac{1}{D} \int_{-D / 2}^{D / 2} f(x) e^{-j 2 \pi \frac{n}{D} x} d x,
$$

$\mathrm{D}$ is the period of the structure, and $\mathrm{n}$ is the $\mathrm{n}_{\mathrm{th}}$ order of diffraction. From this equation, we can see that a periodical structure can be represented by a series of plane waves whose diffraction angle is related to structure period and the weight function for each order is related to aperture ratio. In this experiment we can measure the light spots position and intensity in Fig. 2(b) to get those information. For our SLM, the diffraction angle is 0.0817 rad and intensity ratio of zero to first order is 0.0031 , and we can obtain that the period is $6.5 \mu \mathrm{m}$ and aperture ratio is $90.2 \%$ from this experiment.
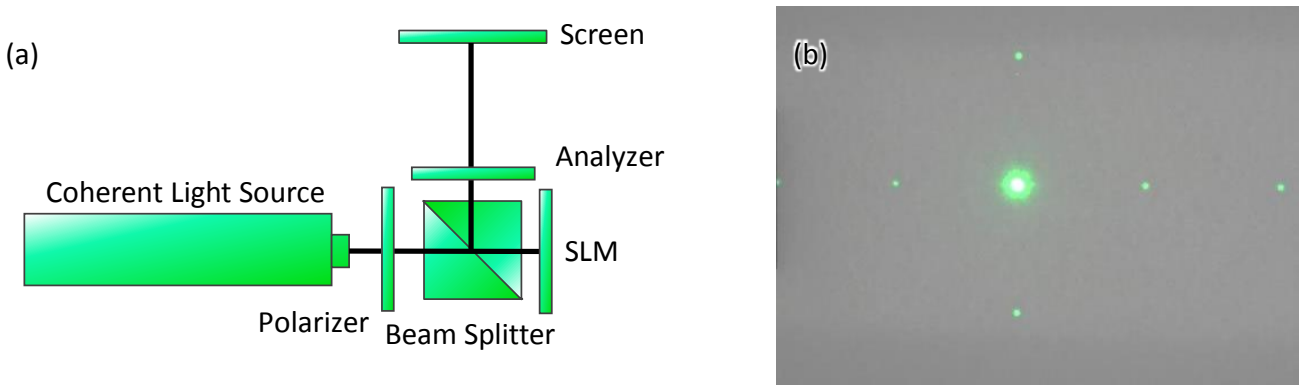

Fig. 2. (a) Experiment setup. (b) Diffracted light spot.

In experiment 2, students will learn how to use SLM as an amplitude modulator. The optical setup is shown in Fig. 3(a), a plane wave passes through SLM with two polarizer controlling input and output polarization state, an imaging lens behind the SLM forms the SLM image onto screen. The transmittance of this system is ${ }^{6,8}$

$$
\mathrm{T}=\cos ^{2} \chi-\sin 2(\beta-\chi) \sin (2 \beta) \sin ^{2}\left[\frac{\delta}{2}\right],
$$

where $\chi$ is the angle between polarizer and analyzer, $\beta$ is the angle between the polarizer and LC director, and $\delta$ is the phase retardation related to voltage applied. With a cross polarized configuration which $\chi$ equal to 90 degree and $\beta$ equals to 45 degree. The transmittance becomes

$$
\mathrm{T}_{\mathrm{A}}=\sin ^{2}\left[\frac{\delta}{2}\right] .
$$

We can get an amplitude modulator with sinusoidal response by this configuration. Fig. 3(b) shows the sinusoidal intensity variation by different grayscale input from computer. 

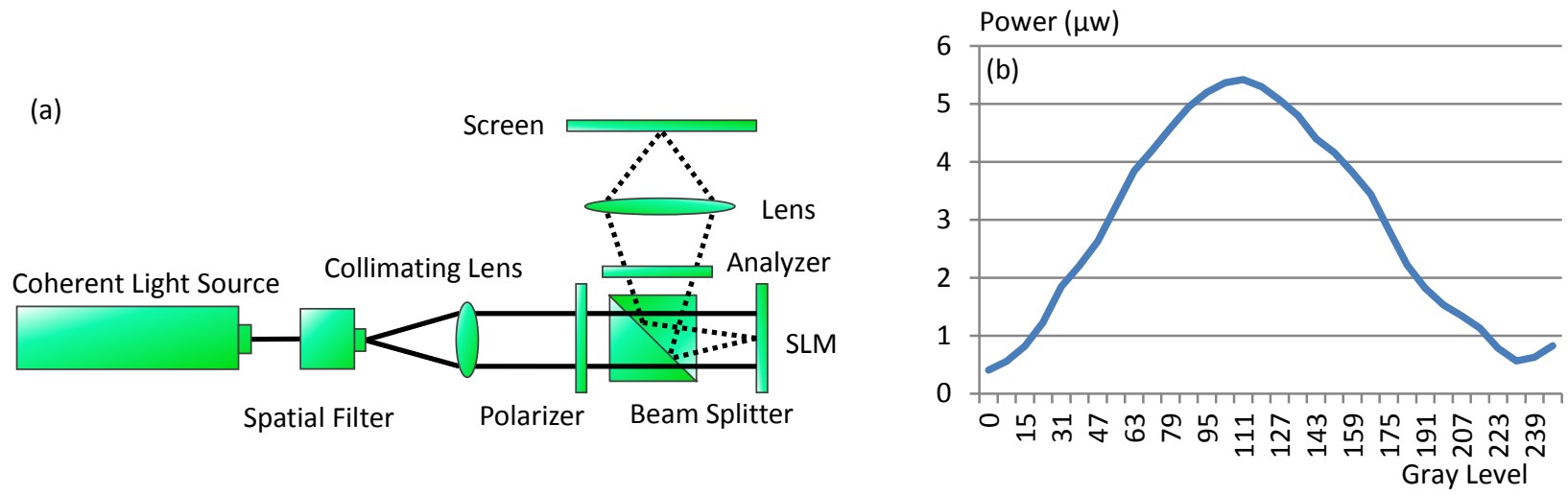

Fig. 3. (a) Experiment setup. (b) Intensity variation with different gray level input.

Experiment 3 is phase modulator, we use the same optical setup as experiment 2 but change the polarizer and analyzer direction to align with LC director, it means $\chi=\beta=0$. As a result, the transmittance is always 1, there is no amplitude variation at this configuration, only phase varies with input signal. We will examine the phase modulation capability by interference, therefore, we add one more mirror for reference light, new setup is shown in Fig. 4(a). We adjust the tilt angle of Mirror to get a horizontal interference and then put a black/white pattern as shown in fig 4(b) to the SLM, each gray level represent different phase difference. The white parts will gradually changes its gray level from 0 to 255 , and the interference pattern will be shifted as equation below

$$
I=2 I_{0}[1+\cos (k \sin \theta y-\phi)]
$$

where $k$ is the wavenumber, $\theta$ is the angle between two plane wave reflected from SLM and mirror, $\phi$ is the phase difference between these two waves. The phase shift is in proportion to interference shift, thus we can measure the phase difference by the shifted interference.

(a)

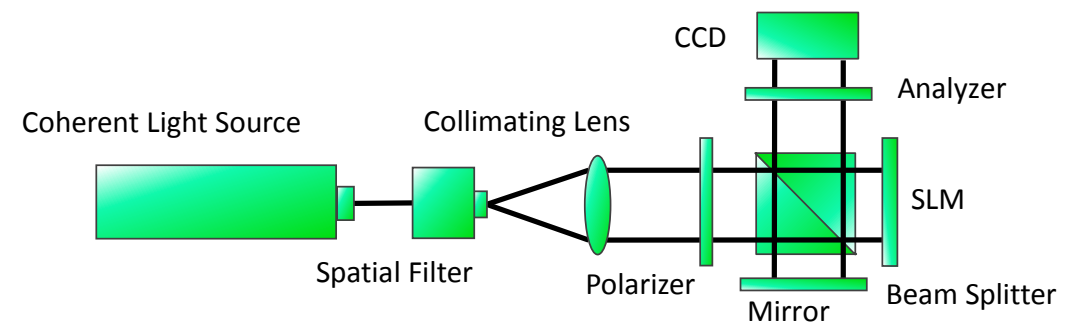

(b)

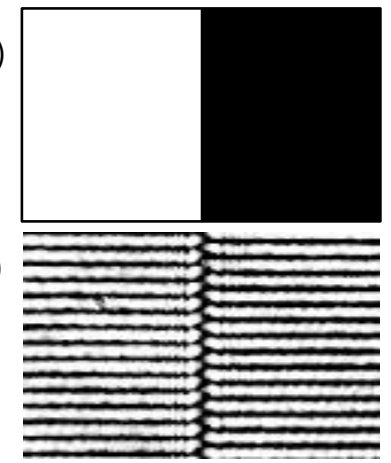

Fig. 4. (a) Experiment setup. (b) Input pattern. (c) Shifted interference.

The last experiment in this section is wavefront modulation, students have known how to use SLM as a phase modulator in previous experiment, and they will learn how to modulate the incident plane wave to converge, diverge, and orientates to other directions. The plane wavefront and spherical wavefront in the SLM plane(x-y plane) can be expressed as following equations

$$
\begin{gathered}
e^{i \frac{2 \pi}{\lambda} x \sin \theta}, \\
e^{i \frac{\pi}{\lambda z_{0}}\left(x^{2}+y^{2}\right)},
\end{gathered}
$$

plane wave

spherical wave (5)

where $\theta$ is the angle between $\mathrm{z}$ axis and propagation direction, $z_{0}$ is the distance from diverging or converging point to the SLM plane. We can directly get the phase information by substituting pixels position(x, y) data of SLM, each pixel will have its phase information and each phase data corresponds to gray level input from computer, the resulting plot are shown in Fig 5(b) and Fig 5(c). The optical system is shown in Fig 5(a), Laser is expanded to illuminate the SLM and two polarizer has the same direction as LC director. Linear polarized plane wave takes phase data from SLM and then wavefront is changed. We can see the result of propagation of modulated wavefront on the screen is shown in Fig. $5(\mathrm{e}) \sim(\mathrm{f})$. 


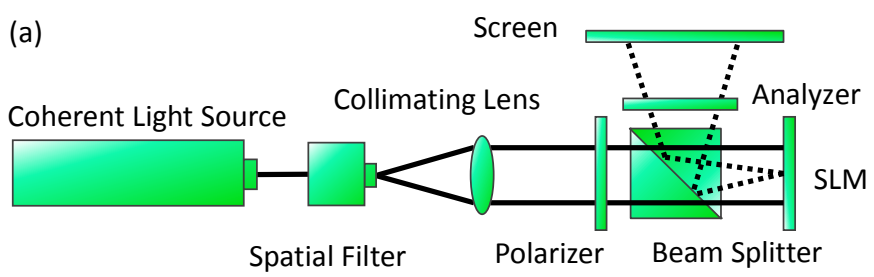

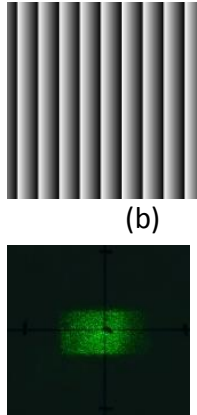

(d)

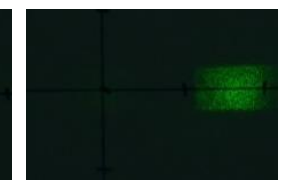

(e) (c)

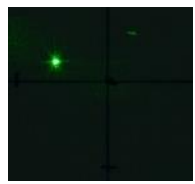

(f)

Fig. 5. (a) Experiment setup. (b)(c) Diffraction pattern for tilt plane wave and converging spherical wave. (d) Reflected plane wave. (e) Tilted plane wave. (f) Converging point.

\section{WAVE OPTICS}

In previous section, those experiments let students familiar with SLM and learn how to operate it with optical components. In this section, SLM will be applied to traditional optical experiments by providing phase grating and amplitude mask. We try to link SLM with traditional experiments and show some idea of applying SLM to education. The first experiment in this section is interference and diffraction, we use the same concept of Young's double-slit experiment, but we use SLM as the tunable slit. SLM is an active optical component which can dynamically change the slit's size and spacing between two slits, therefore, we can easily compare diffraction result by switching slit pattern in computer. The optical setup is shown in Fig 6(a), expanded laser beam take the amplitude data from SLM, two polarizers are cross polarized to get an amplitude modulator, and lens behind the analyzer collect the diffraction caused by amplitude grating to the screen. The resulting interference patter for single slit and double slit are shown in Fig. 6(b) and Fig. 6(c).

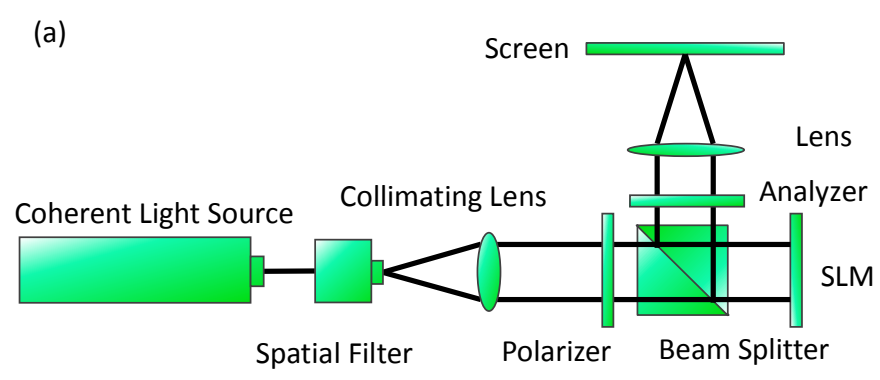

(b)

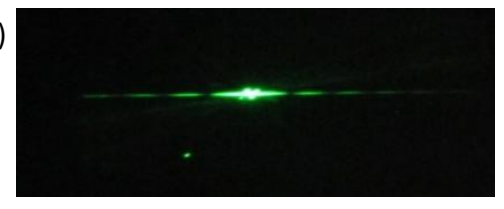

(c)

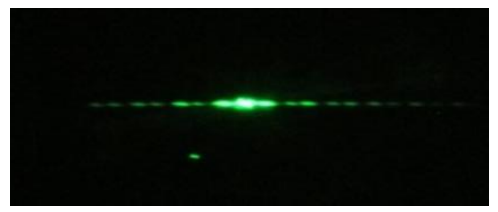

Fig. 6. (a) Experiment setup

(b) Single slit

(c) Double slit(wide slit) interference pattern

Next experiment in this section is Michelson interferometer, we've used this setup in previous section to see the phase modulation by shifted interference pattern. We'll use this setup again but remove the imaging lens as shown in Fig. 7(a). It's a typical Michelson interferometer, but we replace one mirror by SLM as the object wave and light reflected from mirror is the reference wave. We can modulate the object wave reflected from SLM just as what we've done in wavefront modulation. Then object wave and reference wave propagates in the same direction by beam splitter and interfere. CCD behind the analyzer capture the interference as show in Fig.7(b) and 7(c). Fig. 7(b) shows the interference by spherical and plane wave, Fig. 7(c) shows the interference by two plane waves. 
(a)

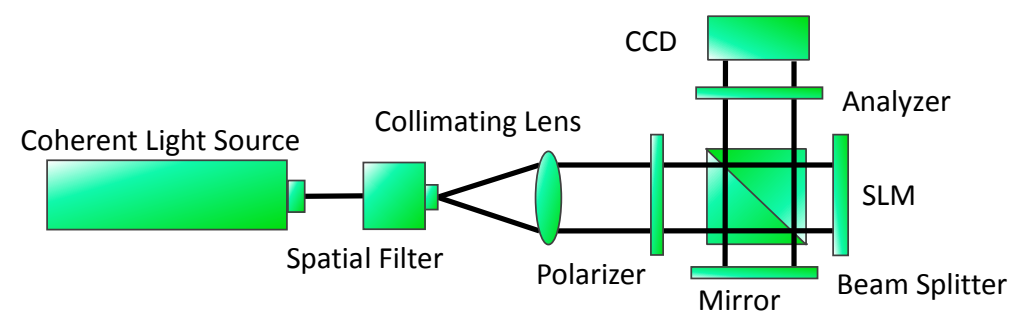

(b)

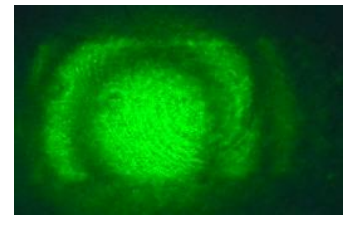

(c)

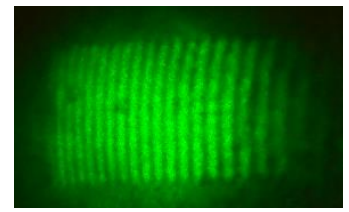

Fig. 7. (a) Experiment setup

(b) Spherical wave and plane wave

(c) Two plane wave interference pattern

The last experiment in section 2 is dispersion, we use LED as the light source and optical setup is shown in Fig. 8(a). An iris is in the image plane of lens 1 in order to get a better resolution, lens 2 is collimating lens for light source image in the iris, and then we put blazed grating as shown in Fig. 5(b) to SLM, the light with different wavelength will be diffracted to different direction and collected by cylindrical lens. The diffraction angle will follow this equation

$$
d \sin \theta_{\mathrm{m}}=\mathrm{m} \lambda
$$

where $d$ is the period of blazed grating, $\theta_{\mathrm{m}}$ is the angle of $\mathrm{m}_{\mathrm{th}}$ diffraction order, and $\lambda$ is the wavelength. Students can find the wavelength by its diffraction angle. The dispersion image is shown in Fig. 8(b).

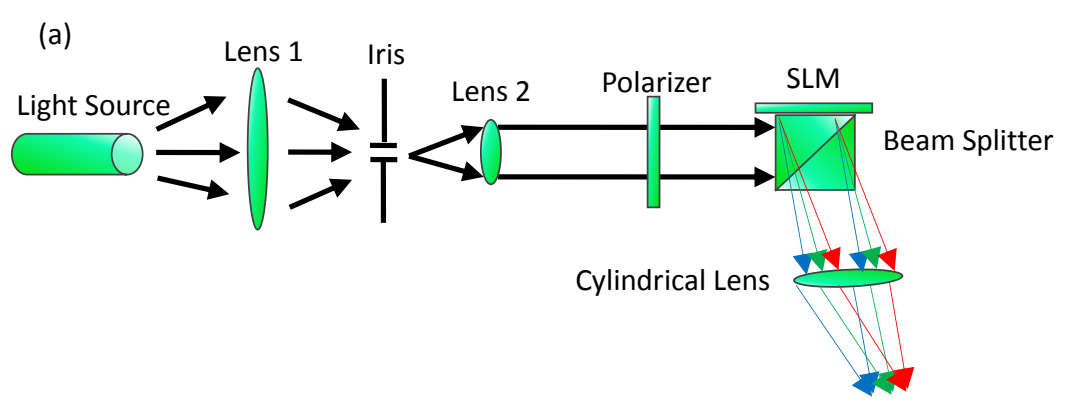

(b)

Fig. 8. (a) Experiment setup

(b) Dispersion of LED light from smart phone's flash light

\section{FOURIER OPTICS}

LCoS SLM has pixelated structure with high aperture ratio and smaller pixel size than other SLM devices, these properties make LCoS SLM good for Fourier experiments which use Fresnel and Fraunhofer diffraction as background knowledge. Because diffraction phenomenon is usually not obvious, we need digital patterns with pixel size near the wavelength range and high reflectivity to get a clear diffraction result.

The first experiment in the last section is signal processing. This experiment is basically a 4-f system, but it becomes reflective type for LCoS SLM. The optical setup is shown in Fig. 9(a), coherent light source was expanded by spatial filter and collimating lens, and then plane wave illuminates the grid pattern as shown in Fig. 9(b), lens behind the pattern transform it into spectrum onto SLM whose function is a filter in this experiment. The spectrum of grid pattern can be obtained by Fourier transform as following equation

$$
\begin{aligned}
\mathcal{F}\{\mathrm{u}(\mathrm{x}, \mathrm{y})\} & =\mathcal{F}\left\{\operatorname{comb}\left(\frac{\mathrm{x}}{\mathrm{D}}\right) * \operatorname{rect}\left(\frac{\mathrm{x}}{\mathrm{d}}\right) \cdot \operatorname{comb}\left(\frac{\mathrm{y}}{\mathrm{D}}\right) * \operatorname{rect}\left(\frac{\mathrm{y}}{\mathrm{d}}\right)\right\} \\
& =\operatorname{comb}\left(\mathrm{Df}_{\mathrm{x}}\right) \cdot \operatorname{sinc}\left(\mathrm{df}_{\mathrm{x}}\right) \cdot \operatorname{comb}\left(\mathrm{Df}_{\mathrm{y}}\right) \cdot \operatorname{sinc}\left(\mathrm{df}_{\mathrm{y}}\right),
\end{aligned}
$$

where $\mathcal{F}$ is the Fourier transform, $\mathrm{D}$ is the spacing between each line, and $\mathrm{d}$ is the width of each line. This shows that there are a lot of light spot with sinc function as envelope signal on SLM plane. We input a slit patter into SLM, and all the high frequency in vertical axis is blocked, only spectrum in horizontal line is preserved. The imaging lens behind the SLM will transform it back to image onto CCD, the new image is shown in Fig. 9(c). 
(a)

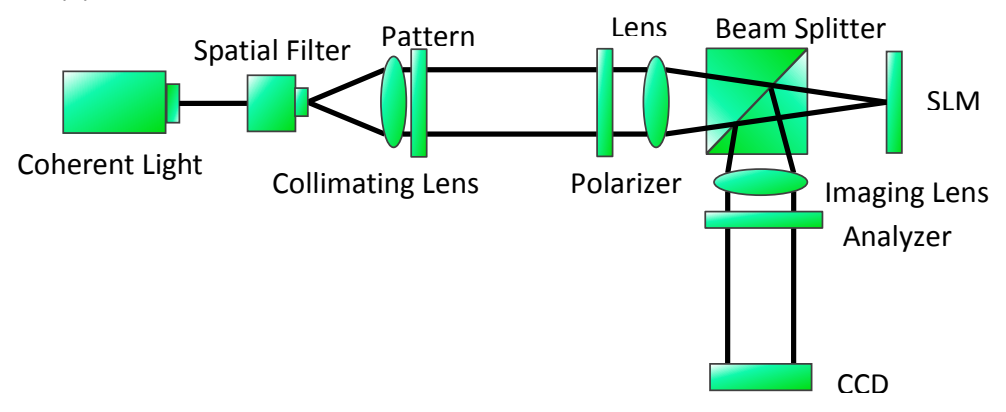

(b)

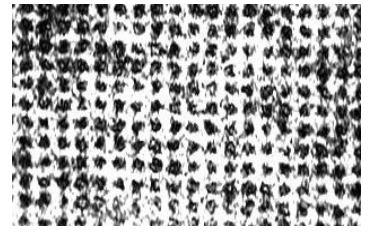

(c)

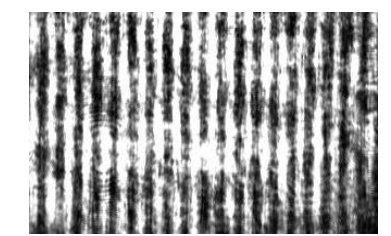

Fig. 9. (a) Experiment setup $\quad$ (b) Original grid pattern $\quad$ (c) Original pattern without high frequency in vertical axis

Next experiment will see the self-imaging phenomenon which is called Talbot image. This happens when the object is a periodical structure and light propagates within a short distance. A periodical structure can be stated as follow ${ }^{5}$

$$
\mathrm{t}_{\mathrm{a}}(\mathrm{x}, \mathrm{y})=\frac{1}{2}\left[1+m \cos \left(2 \pi \frac{\mathrm{x}}{\mathrm{L}}\right)\right]
$$

where $\mathrm{L}$ is the period and it varies along $\mathrm{x}$ axis. The Fresnel diffraction result of this periodical pattern is

$$
\mathrm{I}(\mathrm{u}, \mathrm{v})=\frac{1}{4}\left[1+2 m \cos \left(\frac{\pi \lambda \mathrm{z}}{\mathrm{L}^{2}}\right) \cos \left(\frac{2 \pi \mathrm{u}}{\mathrm{L}}\right)+m^{2} \cos ^{2}\left(\frac{2 \pi \mathrm{u}}{\mathrm{L}}\right)\right],
$$

where $\mathrm{z}$ is propagating distance. When $\mathrm{z}=2 \mathrm{~nL}^{2} / \lambda$, it becomes original image without any lens and this will repeat several times. These images are called Talbot images as shown in Fig. $10(\mathrm{~b})$. When $z=\left(n-\frac{1}{2}\right) \mathrm{L}^{2} / \lambda$, the image has twice frequency of the original image and contrast is reduced. This is called Talbot subimage as shown in Fig. 10(c). The optical system is shown in Fig. 10(a), plane wave bring the periodical structure image from SLM and reflected onto CCD and then CCD is moved back and forth to find the Talbot image and Talbot subimage.

(a)

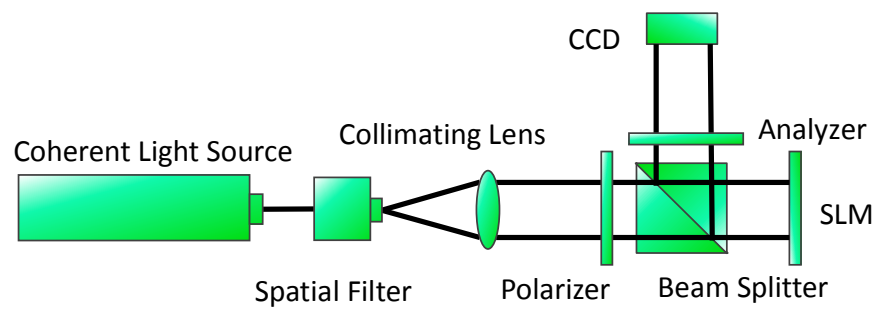

Fig. 10. (a) Experiment setup (b) Talbot image (b)

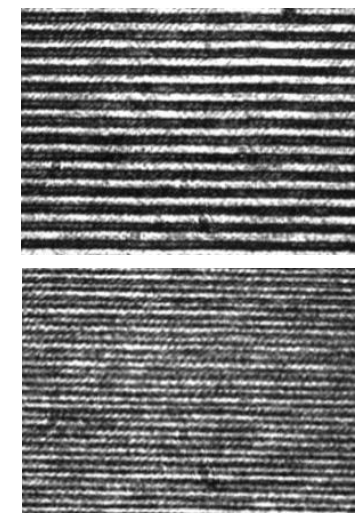

(c) Talbot subimage

The last experiment for LCoS SLM is PSDH (Phase Shifting Digital Holography). An object wave can be recorded by recording one set interference pattern. In PSDH we record 4 interference patterns each has different phase shift $\left(0, \frac{\pi}{2}, \pi, \frac{3 \pi}{2}\right)$ in reference wave. By the following equation we can get the original wave information ${ }^{9}$

$$
\mathrm{O}(\mathrm{x}, \mathrm{y})=\frac{1}{4|R|}\left[\left(\mathrm{I}_{0}-\mathrm{I}_{\pi}\right)+i\left(\mathrm{I} \pi / 2-\mathrm{I}_{3 \pi / 2}\right)\right] \text {, }
$$

where $\mathrm{R}$ is the reference wave, $\mathrm{I}$ is interference of object and reference wave, and subscript 0 means the reference interference, other value mean the phase shift in reference wave. Therefore, we use Michaelson interferometer again and SLM is used to give phase retardation to the reference wave by giving different gray level input. The optical setup and interference set is shown in Fig 11(a) and (b), and then we use equation above and Fresnel diffraction to calculate the object wave, result is shown in Fig. 11(c). 
(a)

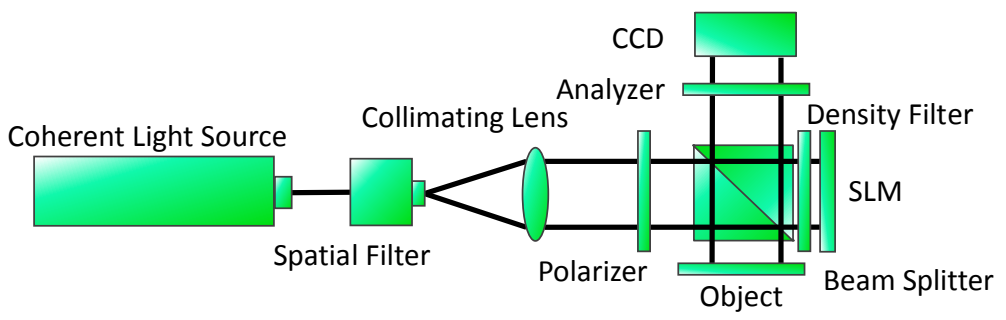

(b)

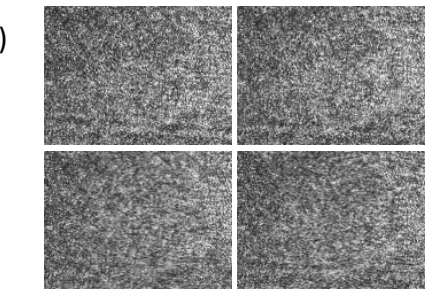

(c)

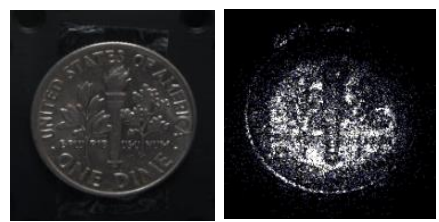

Fig. 11. (a) Experiment setup $\quad$ (b) One set of interference $\quad$ (c) Original image(left) and simulated image(right)

\section{SOFTWARE}

The operation of LCoS SLM in our educational kit is like a micro display, we calculate phase information on SLM plane, convert phase data into gray level data, draw the gray level pattern, and export patter from computer to SLM via HDMI cable. But drawing pattern is usually a hard work for students because they need to calculate phase data and convert this data into pattern. Therefore, we also designed software for these experiments. The concept of the software is pretty simple, it's a pattern generator which generates basic diffraction pattern to SLM and dynamically responses to input parameters. The software skin is shown in Fig. 12, two windows at the bottom are description and diagram of optic system, and windows on the top are calculated grating and parameter slide bar which controls the diffraction angle, rotation, focal length, and so on. Users can drag the slide bar to change the pattern and send it to SLM automatically. Software also provides macro for user to define their experiments including mathematical formula of diffraction grating, parameters, description, and diagram.

\section{CONCLUSION}

In conclusion, SLM is a powerful optical device and it has many applications, but those applications needs base knowledge to a certain degree, hence there is a gap in learning SLM. Therefore these experiments do not have complicated optic experiments and focus on two things, "how to operate" and "what gratings can do". We hope this can help students easily start to learn. In future work, we'll try to develop educational kit further by simplify SLM's application and introduce these applications to SLM users.

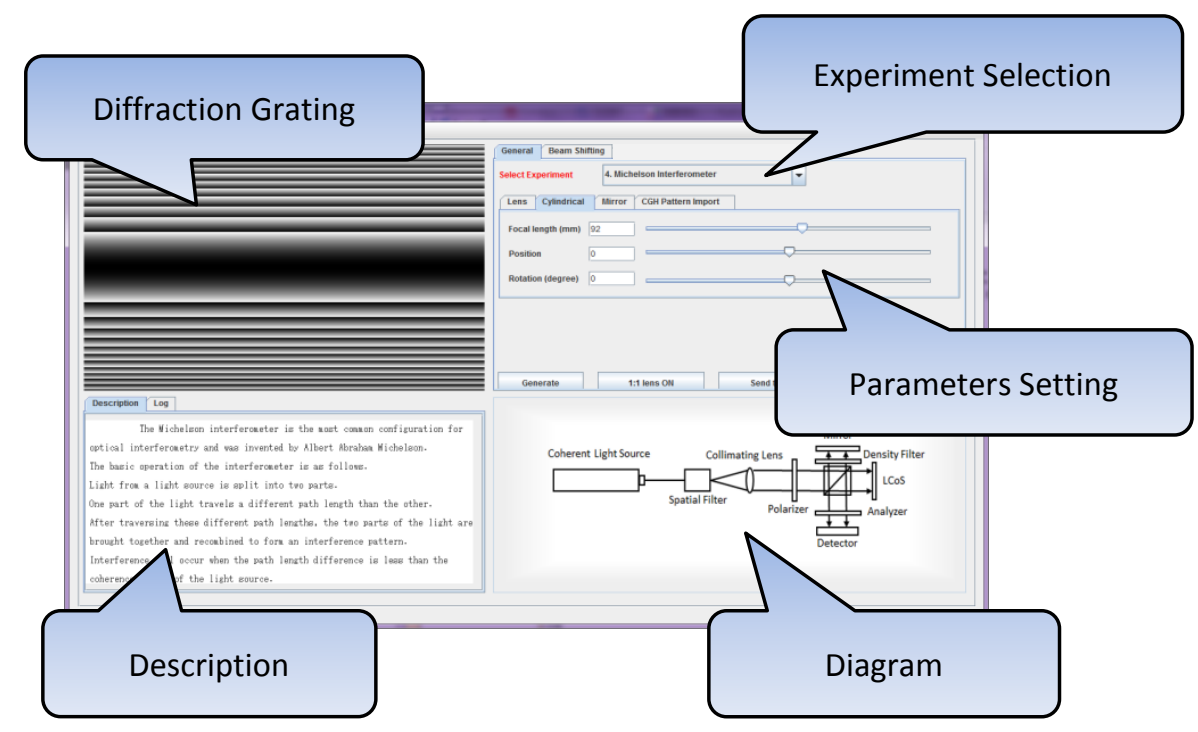

Fig. 12. Software skin 


\section{REFERENCES}

[1] Chung, C.Y., Cho, K.C., Chang, C.C., Lin, C.H., Yen, W.C., Chen, S.J. [Adaptive-optics system with liquid-crystal phase- shift interferometer], APPLIED OPTICS, Vol. 45, No. 15, 3409-3414(2006).

[2] Kozacki, T., [Holographic display with tilted spatial light modulator], APPLIED OPTICS, Vol. 50, No. 20, 3580-3588(2011).

[3] Li, S., Wang, Y., Lu, Z., Ding, L., Du, P., Chen, Y., Zheng, Z., Ba, D., Dong, Y., Yuan, H., Bai, Z., Liu, Z., Cui, C., [High-quality near-field beam achieved in a high-power laser based on SLM adaptive beam-shaping system], OPTICS EXPRESS, Vol. 23, No. 2, 681-689(2015).

[4] McManamon, P.F., Bos, P.J., Escuti, M.J., Heikenfeld, J., Serati, S., Xie, H., Watson, E.A., [A Review of Phased Array Steering for Narrow-Band Electrooptical Systems], Proceedings of IEEE, Vol. 97, No. 6, 1078-1096(2009).

[5] Goodman, J. W., [Introduction to Fourier Optics], McGraw-Hill, Singapore (1996).

[6] Wu, S. T., Yang, D. K., [Reflective Liquid Crystal Displays], WILEY SID Series in Display Technology, England(2001)

[7] Hecht, E., [Optics], Addison Wesley, San Francisco (2002).

[8] Yariv, A., Yeh, P., [Optical Waves in Crystals], WILEY INTER-SCIENCE, Canada (2003).

[9] Yamaguchi, I., Zhang, T., [Phase-shifting digital holography], Optics Letters, Vol. 22, Issue 16, 1268-1270(1997). 